УДК 517.926, 517.977.1

\title{
Differential Controllability of Linear Systems of Differential-algebraic Equations
}

Pavel S. Petrenko*

Matrosov Institute for System Dynamics and Control Theory SB RAS Lermontov, 134, Irkutsk, 664033,

Russian

Received 05.08.2016, received in revised form 06.03.2017, accepted 10.04.2017

Linear controllable system of first order ordinary differential equations is considered. The system is unresolved with respect to the derivative of the unknown function and it is identically degenerate in the domain. An arbitrarily high unresolvability index is admitted. Differential controllability of the system is investigated under assumptions that ensure the existence of a global structural form that separates "algebraic" and "differential" subsystems.

Keywords: differential-algebraic equations, differential controllability, full controllability. DOI: $10.17516 / 1997-1397-2017-10-3-320-329$

\section{Introduction and preliminaries}

Let us consider the system of ordinary differential equations (ODEs)

$$
A(t) x^{\prime}(t)=B(t) x(t)+U(t) u(t), \quad t \in I=[0,+\infty),
$$

where $A(t), B(t)$ are known $(n \times n)$-matrices, $U(t)$ is known $(n \times l)$-matrix, $x(t)$ is unknown $n$-dimensional function of state, $u(t)$ is $l$-dimensional function of control. It is assumed that $\operatorname{det} A(t) \equiv 0$.

Such systems are called differential-algebraic equations (DAEs). The measure of unresolvability for DAEs with respect to the derivative is an integer $r: 0 \leqslant r \leqslant n$, which is called index $[1,2]$.

There are a lot of various concepts of controllability in the theory of DAEs. The property of the full controllability (see., eg, [3, 4]) was first introduced and investigated for the DAEs with constant coefficients in [5]. The most important concepts of $R$-controllability and impulse controllability were first introduced for the DAEs with constant coefficients and regular matrix pencil [6]. The resulting algebraic criteria are used in the analysis of the problem of minimizing a quadratic functional on solutions of the linear DAEs. Conditions of $R$-controllability for the linear DAEs with infinitely differentiable coefficients were obtained [7]. Conditions of $R$-controllability for the DAEs with variable coefficients and arbitrarily high unresolvability index were obtained [8]. Differential controllability of systems resolved with respect to the derivative (ODEs) was investigated [3].

In this paper we investigate differential controllability of linear DAEs systems with variable coefficients. The necessary and sufficient conditions of the differential controllability of such systems are obtained. The analysis is carried out under assumptions that ensure the existence of a global structural form that separates "algebraic" and "differential" subsystems. They have the same solutions as the original system [8-10].

*petrenko_p@mail.ru

(c) Siberian Federal University. All rights reserved 


\section{Equivalent structural form}

Let us introduce the following matrices for system (1.1)

$$
\begin{gathered}
\mathrm{D}_{r, z}(t)=\left(\begin{array}{cccc}
C_{1}^{1} A(t) & O & \ldots & O \\
C_{2}^{1} A^{\prime}(t)+C_{2}^{2} B(t) & C_{2}^{2} A(t) & \ldots & O \\
\vdots & \vdots & \ddots & \vdots \\
C_{r}^{1} A^{(r-1)}(t)+C_{r}^{2} B^{(r-2)}(t) & C_{r}^{2} A^{(r-2)}(t)+C_{r}^{3} B^{(r-3)}(t) & \ldots & C_{r}^{r} A(t)
\end{array}\right), \\
\mathrm{D}_{r, y}(t)=\left(\begin{array}{c}
C_{0}^{0} A(t) \\
\left(\begin{array}{c}
C_{1}^{0} A^{\prime}(t)+C_{1}^{1} B(t) \\
\vdots \\
C_{r}^{0} A^{(r)}(t)+C_{r}^{1} B^{(r-1)}(t)
\end{array}\right) \\
\mathrm{D}_{r, z}
\end{array}\right), \quad \mathrm{D}_{r, x}(t)=\left(\begin{array}{cc}
B \\
(t) & \mathrm{D}_{r, y}
\end{array}\right) .
\end{gathered}
$$

They have dimensions $n r \times n r, n(r+1) \times n(r+1)$ and $n(r+1) \times n(r+2)$, respectively. From this point on $C_{i}^{j}=\frac{i !}{j !(i-j) !}$ are binomial coefficients, $O$ is the null matrix of appropriate size, $\bar{B}(t)=$ colon $\left(B(t), B^{\prime}(t), \ldots, B^{(r)}(t)\right)^{\dagger}$.

Let us suppose that condition $\operatorname{rank} \mathrm{D}_{r, z}(t)=\rho=$ const $\forall t \in I$ holds for some $r(0 \leqslant r \leqslant n)$ and there is non-special minor $\forall t \in I$ of the order $n(r+1)$ in the matrix $\mathrm{D}_{r, x}(t)$. This minor includes $\rho$ columns of the matrix $\mathrm{D}_{r, z}$ and $n$ first columns of the matrix $\mathrm{D}_{r, y}$. Such minor is called resolving.

Let us assume that we know exactly which columns of the matrix $\mathrm{D}_{r, x}(t)$ are included into the resolving minor. We delete $n-d$ columns of the matrix $\bar{B}(t)$ which are not included in this minor, where $d=n r-\rho$. After the appropriate column permutation of $\mathrm{D}_{r, x}(t)$ we obtain the matrix

$$
\Lambda_{r}(t)=\mathrm{D}_{r, x}(t) \operatorname{diag}\left(Q^{-1}\left(\begin{array}{c}
O \\
E_{d}
\end{array}\right), Q^{-1}, \ldots, Q^{-1}\right) \ddagger
$$

where $E_{d}$ is the identity matrix of order $d, Q$ is $(n \times n)$-permutation matrix ${ }^{\S}$.

Matrix $Q^{-1}$ is constructed as follows. Let us denote the numbers of columns of $\bar{B}(t)$ by $i_{1}, i_{2}, \ldots, i_{d}$ and $i_{d+1}, i_{d+2}, \ldots, i_{n}$ as the numbers of columns of $\bar{B}(t)$. They are included and not included in the resolving minor, respectively. Being left multiplied by matrix $\bar{B}(t)$, matrix $Q^{-1}$ puts every $\left(i_{d+k}\right)$-th column $(k=\overline{1, n-d})$ into $k$-th place and every $\left(i_{j}\right)$-th column $(j=\overline{1, d})$ into the place with number $n-d+j$ in the matrix $\bar{B}(t)$. Matrix $Q^{-1}$ is invertible and it consists of zeros and $n$ ones, wherein ones are the elements with indices $\left(i_{d+k}, k\right)$ and $\left(i_{j}, n-d+j\right)$.

Lemma 1. Let us assume that

1) $A(t), B(t), U(t), u(t) \in \mathbf{C}^{r}(I)$;

2) $\operatorname{rank} \mathrm{D}_{r, z}(t)=\rho=$ const $\forall t \in I$;

3) there is the resolving minor in matrix $\mathrm{D}_{r, x}(t)$.

Then there exists linear differential operator

$$
\mathcal{R}=R_{0}(t)+R_{1}(t) \frac{d}{d t}+\ldots+R_{r}(t)\left(\frac{d}{d t}\right)^{r}
$$

$$
{ }^{\dagger} \operatorname{colon}\left(x_{1}, x_{2}, \ldots, x_{n}\right)=\left(\begin{array}{c}
x_{1} \\
x_{2} \\
\vdots \\
x_{n}
\end{array}\right)
$$

${ }^{\ddagger}$ The notation $\operatorname{diag}\left(A_{1}, A_{2}, \ldots, A_{s}\right)$ denotes quasi-diagonal matrix with the blocks listed in parenthesis on the main diagonal. Other elements are zero.

${ }^{\S}$ See [11] about row and column permutation matrix. 
with continuous coefficients $R_{j}(t)(j=\overline{0, r})$ that converts system (1.1) into the form

$$
\begin{aligned}
& x_{1}^{\prime}(t)=J_{1}(t) x_{1}(t)+\mathcal{H}(t) \bar{u}(t), \\
& x_{2}(t)=J_{2}(t) x_{1}(t)+\mathcal{G}(t) \bar{u}(t),
\end{aligned}
$$

where colon $\left(x_{1}(t), x_{2}(t)\right)=Q x(t), \bar{u}(t)=\operatorname{colon}\left(u(t), u^{\prime}(t), \ldots, u^{(r)}(t)\right)$,

$$
\begin{aligned}
& \left(\begin{array}{c}
\mathcal{G}(t) \\
\mathcal{H}(t)
\end{array}\right)=\left(\begin{array}{cccc}
G_{0}(t) & G_{1}(t) & \ldots & G_{r}(t) \\
H_{0}(t) & H_{1}(t) & \ldots & H_{r}(t)
\end{array}\right)=\left(\begin{array}{lllll}
R_{0}(t) & R_{1}(t) & \ldots & \left.R_{r}(t)\right) \mathcal{P}_{r}[U(t)]
\end{array}\right. \\
& \mathcal{P}_{r}[U(t)]=\left(\begin{array}{cccc}
C_{0}^{0} U(t) & O & \ldots & O \\
C_{1}^{0} U^{\prime}(t) & C_{1}^{1} U(t) & \ldots & O \\
\vdots & \vdots & \ddots & \vdots \\
C_{r}^{0} U^{(r)}(t) & C_{r}^{1} U^{(r-1)}(t) & \ldots & C_{r}^{r} U(t)
\end{array}\right) \\
& \left(\begin{array}{cc}
J_{2}(t) & E_{d} \\
J_{1}(t) & O
\end{array}\right)=\left(\begin{array}{llll}
R_{0}(t) & R_{1}(t) & \ldots & \left.R_{r}(t)\right) \bar{B}(t) Q^{-1}
\end{array}\right.
\end{aligned}
$$

Matrices $R_{j}(t)$ are uniquely determined by the resolving minor as

$$
\left(\begin{array}{llll}
R_{0}(t) & R_{1}(t) & \ldots & R_{r}(t)
\end{array}\right)=\left(\begin{array}{lllll}
E_{n} & O & \ldots & O
\end{array}\right) \Lambda_{r}^{\top}(t)\left(\Lambda_{r}(t) \Lambda_{r}^{\top}(t)\right)^{-1} .
$$

Definition 1. With a given control function $u(t) n$-dimensional vector function $x(t) \in \mathbf{C}^{1}(I)$ is the solution of (1.1) if it identically satisfies this system on I.

Theorem 1. Let us assume that

1) $A(t), B(t), U(t), u(t) \in \mathbf{C}^{2 r+1}(I)$;

2) $\operatorname{rank} \mathrm{D}_{r, z}(t)=\rho=$ const $\forall t \in I$;

3) there is the resolving minor in matrix $\mathrm{D}_{r, x}(t)$;

4) $\operatorname{rank} \mathrm{D}_{r+1, y}(t)=\operatorname{rank} \mathrm{D}_{r, y}(t)+n \forall t \in I$.

Then every solution of (1.1) is the solution of (2.3), (2.4) and vice versa.

Definition 2. System (2.3), (2.4) is called the equivalent form of DAE (1.1).

Let us define the initial conditions

$$
x\left(t_{0}\right)=x_{0},
$$

where $t_{0} \in I, \quad x_{0} \in \mathbf{R}^{n}$ is a given vector.

Theorem 1 provides a criterion for the existence and uniqueness of solution to problem (1.1), (2.7).

Corollary 1. Let us suppose that all the assumptions of Theorem 1 are satisfied. Then problem (1.1), (2.7) is solvable if and only if

$$
x_{2,0}=J_{2}\left(t_{0}\right) x_{1,0}+\mathcal{G}\left(t_{0}\right) \bar{u}\left(t_{0}\right),
$$

where $\left(\begin{array}{l}x_{1,0} \\ x_{2,0}\end{array}\right)=Q x_{0}$. Moreover, if a solution to problem (1.1), (2.7) exists then it is unique.

Definition 3. Initial condition (2.7) which satisfies (2.8) is called consistent with system (1.1) in the point $t_{0}$.

There are proofs of all results of this section [10]. 


\section{Differential controllability}

Definition 4. System (1.1) is called completely controllable on interval $T=\left[t_{0}, t_{1}\right] \subset I$ if for any $x_{0}, x_{1} \in \mathbf{R}^{n}$ there exists control function $u(t)$ such that the appropriate solution $x(t)$ of $D A E$ (1.1) satisfies conditions $x\left(t_{0}\right)=x_{0}, x\left(t_{1}\right)=x_{1}$.

Definition 5. System (1.1) is called differentially controllable on the interval $T$ if it is completely controllable on any set $\left[\tau_{0}, \tau_{1}\right] \subset T$.

\subsection{Non-stationary systems}

In this section we prove necessary and sufficient conditions for the differential controllability of DAE (1.1).

Theorem 2. Let us suppose that all the assumptions of Theorem 1 are satisfied. System (1.1) is differentially controllable on $T$ if and only if the following conditions are satisfied:

1) $\operatorname{rank} \mathcal{G}(t)=d \forall t \in T$;

2) $\forall h \in \mathbf{R}^{n-d}: h \neq 0, \quad h^{\top} X^{-1}(t) \mathcal{H}(t) \not \equiv 0$ for almost all $t$, i.e. for all points $t \in T$ except some sets with zero measure $\boldsymbol{\Phi}^{\mathbb{T}}$ Herein $X(t)$ is $(n-d) \times(n-d)$ fundamental matrix of the homogeneous system $x_{1}^{\prime}(t)=J_{1}(t) x_{1}(t)$.

Proof. Necessity. Let system (1.1) be differentially controllable on $T$. This means that it is completely controllable on any set $\left[\tau_{0}, \tau_{1}\right] \subset T$. It follows from Theorem 1 that system $(2.3),(2.4)$ has the same property. Then, any solution of (2.3), (2.4) should satisfy the following relations

$$
\begin{gathered}
x_{2}\left(\tau_{0}\right)-J_{2}\left(\tau_{0}\right) x_{1}\left(\tau_{0}\right)=\mathcal{G}\left(\tau_{0}\right) \bar{u}\left(\tau_{0}\right), \\
x_{2}\left(\tau_{1}\right)-J_{2}\left(\tau_{1}\right) x_{1}\left(\tau_{1}\right)=\mathcal{G}\left(\tau_{1}\right) \bar{u}\left(\tau_{1}\right), \\
X^{-1}\left(\tau_{1}\right) x_{1}\left(\tau_{1}\right)-x_{1}\left(\tau_{0}\right)=\int_{\tau_{0}}^{\tau_{1}} X^{-1}(t) \mathcal{H}(t) \bar{u}(t) d t .
\end{gathered}
$$

If relations (3.1), (3.2) are fulfilled for all left-hand sides then we have completeness of the ranks of matrices $\mathcal{G}\left(\tau_{0}\right)$ and $\mathcal{G}\left(\tau_{1}\right)$, respectively. Since points $\tau_{0}, \tau_{1}\left(\tau_{1}>\tau_{0}\right)$ are arbitrarily selected, it implies completeness of the rank of the matrix $\mathcal{G}(t) \forall t \in T$, and leads to condition 1) of the Theorem.

Let us assume that condition 2) is not satisfied, i.e. there exists a nonzero vector $h \in \mathbf{R}^{n-d}$ such that $h^{\top} X^{-1}(t) \mathcal{H}(t)=0$ on some interval $\left[\tau_{0}^{*}, \tau_{1}^{*}\right] \subset T$. At the same time it follows from equation (3.3) that matrix $\int_{\tau_{0}}^{\tau_{1}} X^{-1}(t) \mathcal{H}(t) \bar{u}(t) d t \neq 0 \quad \forall t \in\left[\tau_{0}, \tau_{1}\right]$ for arbitrary $\tau_{0}, \tau_{1} \in T\left(\tau_{1}>\right.$ $\left.\tau_{0}\right)$ including $\tau_{0}=\tau_{0}^{*}, \tau_{1}=\tau_{1}^{*}$. We have a contradiction. In this way,

$$
h^{\top} X^{-1}(t) \mathcal{H}(t) \not \equiv 0
$$

for almost all $t$.

Sufficiency. Let us suppose that conditions 1), 2) of the Theorem are satisfied. One needs to show that in this case there exists a vector-function of control $u(t) \in \mathbf{C}^{2 r+1}(T)$ such that equalities (3.1)-(3.3) holds for all left-hand sides.

Let us assume that control function $u(t)$ is a polynomial

$$
u(t)=\sum_{j=0}^{r}\left(\alpha_{j}\left(t-\tau_{0}\right)^{r+1+j}+\beta_{j}\left(t-\tau_{1}\right)^{r+1+j}+\gamma_{j}\left(t-\tau_{0}\right)^{r+1}\left(t-\tau_{1}\right)^{r+1}(t-c)^{s+j}\right),
$$

\footnotetext{
I A set located on interval $[a, b]$ is called a set of zero measure if for every $\varepsilon>0$ it can be covered by a finite
} or countable system of intervals. The sum of the lengths of intervals does not exceed $\varepsilon$. 
where $\alpha_{j}, \beta_{j}, \gamma_{j} \in \mathbf{R}^{n-d}$ are unknown coefficients, $c \notin T$ is constant, and $s>2 r+1$ is some integer.

Then

$$
\begin{gathered}
\bar{u}(t)=\Lambda_{r+1}\left(t-\tau_{0}\right) \operatorname{colon}\left(\alpha_{0}, \ldots, \alpha_{r}\right)+\Lambda_{r+1}\left(t-\tau_{1}\right) \operatorname{colon}\left(\beta_{0}, \ldots, \beta_{r}\right)+ \\
+\Phi\left(t-\tau_{0}, t-\tau_{1}\right) \Lambda_{s}(t-c) \operatorname{colon}\left(\gamma_{0}, \ldots, \gamma_{r}\right)
\end{gathered}
$$

where

$$
\Lambda_{k}(t)=\left(\begin{array}{cccc}
\frac{k !}{k !} t^{k} E_{l} & \frac{(k+1) !}{(k+1) !} t^{k+1} E_{l} & \ldots & \frac{(k+r) !}{(k+r) !} t^{k+r} E_{l} \\
\frac{k !}{(k-1) !} t^{k-1} E_{l} & \frac{(k+1) !}{k !} t^{k} E_{l} & \cdots & \frac{(k+r) !}{(k+r-1) !} t^{k+r-1} E_{l} \\
\vdots & \vdots & \ddots & \vdots \\
\frac{k !}{(k-r) !} t^{k-r} E_{l} & \frac{(k+1) !}{(k+1-r) !} t^{k+1-r} E_{l} & \cdots & \frac{(k+r) !}{k !} t^{k} E_{l}
\end{array}\right),
$$

$k=r+1, \ldots, s$
$\Phi(t, \tau)=$

$$
=\left(\begin{array}{cccc}
C_{0}^{0} \frac{((r+1) !)^{2}(t \tau)^{r+1}}{((r+1) !)^{2}} E_{l} & O & \ldots & O \\
C_{1}^{0}\left(\sum_{i=0}^{1} C_{1}^{i} \frac{((r+1) !)^{2} t^{r+i} \tau^{r+1-i}}{(r+i) !(r+1-i) !}\right) E_{l} & C_{1}^{1} \frac{((r+1) !)^{2}(t \tau)^{r+1}}{((r+1) !)^{2}} E_{l} & \ldots & O \\
\vdots & \vdots & \ddots & \vdots \\
C_{r}^{0}\left(\sum_{i=0}^{r} C_{r}^{i} \frac{((r+1) !)^{2} t^{1+i} \tau^{r+1-i}}{(1+i) !(r+1-i) !}\right) E_{l} & C_{r}^{1}\left(\sum_{i=0}^{r-1} C_{r-1}^{i} \frac{((r+1) !)^{2} t^{2+i} \tau^{r+1-i}}{(2+i) !(r+1-i) !}\right) E_{l} & \ldots & C_{r}^{r} \frac{((r+1) !)^{2}(t \tau)^{r+1}}{((r+1) !)^{2}} E_{l}
\end{array}\right) .
$$

Let us introduce the following designations

$$
\begin{gathered}
g_{1}=x_{2}\left(\tau_{0}\right)-J_{2}\left(\tau_{0}\right) x_{1}\left(\tau_{0}\right), \quad g_{2}=x_{2}\left(\tau_{1}\right)-J_{2}\left(\tau_{1}\right) x_{1}\left(\tau_{1}\right), \quad g_{3}=X^{-1}\left(\tau_{1}\right) x_{1}\left(\tau_{1}\right)-x_{1}\left(\tau_{0}\right) ; \\
\bar{\alpha}=\operatorname{colon}\left(\alpha_{0}, \ldots, \alpha_{r}\right), \quad \bar{\beta}=\operatorname{colon}\left(\beta_{0}, \ldots, \beta_{r}\right), \quad \bar{\gamma}=\operatorname{colon}\left(\gamma_{0}, \ldots, \gamma_{r}\right) .
\end{gathered}
$$

Substituting (3.6) into (3.1) and (3.2), we obtain the system of equations with respect to coefficients $\bar{\alpha}$ and $\bar{\beta}$ :

$$
\begin{aligned}
& \mathcal{G}\left(\tau_{0}\right) \Lambda_{r+1}\left(\tau_{0}-\tau_{1}\right) \bar{\beta}=g_{1}, \\
& \mathcal{G}\left(\tau_{1}\right) \Lambda_{r+1}\left(\tau_{1}-\tau_{0}\right) \bar{\alpha}=g_{2} .
\end{aligned}
$$

It is obvious that for $\tau_{0} \neq \tau_{1}$ matrix $\Lambda_{k}(t)$ is invertible for all $t \in\left[\tau_{0}, \tau_{1}\right]$. Therefore, taking into account condition 1) of the Theorem, we can uniquely determine coefficients $\bar{\alpha}$ and $\bar{\beta}$ :

$$
\begin{aligned}
& \bar{\beta}=\Lambda_{r+1}^{-1}\left(\tau_{0}-\tau_{1}\right) \mathcal{G}\left(\tau_{0}\right)^{\top}\left(\mathcal{G}\left(\tau_{0}\right) \mathcal{G}\left(\tau_{0}\right)^{\top}\right)^{-1} g_{1}, \\
& \bar{\alpha}=\Lambda_{r+1}^{-1}\left(\tau_{1}-\tau_{0}\right) \mathcal{G}\left(\tau_{1}\right)^{\top}\left(\mathcal{G}\left(\tau_{1}\right) \mathcal{G}\left(\tau_{1}\right)^{\top}\right)^{-1} g_{2} .
\end{aligned}
$$

Substituting (3.6), (3.9) and (3.10) into equation (3.3) we obtain the system of equations with respect to $\bar{\gamma}$ :

$$
\begin{gathered}
g_{3}-\int_{\tau_{0}}^{\tau_{1}} X^{-1}(t) \mathcal{H}(t)\left(\Lambda_{r+1}\left(t-\tau_{0}\right) \bar{\alpha}+\Lambda_{r+1}\left(t-\tau_{1}\right) \bar{\beta}\right) d t= \\
=\int_{\tau_{0}}^{\tau_{1}} X^{-1}(t) \mathcal{H}(t) \Phi\left(t-\tau_{0}, t-\tau_{1}\right) \Lambda_{s}(t-c) d t \bar{\gamma} .
\end{gathered}
$$


Matrix $\Phi\left(t-\tau_{0}, t-\tau_{1}\right)$ is invertible on $\left(\tau_{0}, \tau_{1}\right)$ and $\Phi\left(t-\tau_{0}, t-\tau_{1}\right)=O$ in points $t=\tau_{0}$ and $t=\tau_{1}$. Then, by virtue of condition 2) of the Theorem for any nonzero vector $h \in \mathbf{R}^{n-d}$ the relation

$$
h^{\top} X^{-1}(t) \mathcal{H}(t) \Phi\left(t-\tau_{0}, t-\tau_{1}\right) \not \equiv 0 .
$$

is fulfilled. As discussed above, matrix $\Lambda_{s}(t-c)$ is invertible for all $t \neq c$. In this case, it is easy to see that for sufficiently large $s$ the relation

$$
h^{\top} \int_{\tau_{0}}^{\tau_{1}} X^{-1}(t) \mathcal{H}(t) \Phi\left(t-\tau_{0}, t-\tau_{1}\right) \Lambda_{s}(t-c) d t=h^{\top} N \neq 0 \quad \forall t \in\left[\tau_{0}, \tau_{1}\right],
$$

is fulfilled for all nonzero $h \in \mathbf{R}^{n-d}$. This means that matrix $N$ has full row rank.

Thus, the solvability of the equation

$$
\bar{\gamma}=N^{\top}\left(N N^{\top}\right)^{-1} \tilde{g}_{3}
$$

follows from (3.11), where

$$
\tilde{g}_{3}=g_{3}-\int_{\tau_{0}}^{\tau_{1}} X^{-1}(t) \mathcal{H}(t)\left(\Lambda_{r+1}\left(t-\tau_{0}\right) \bar{\alpha}+\Lambda_{r+1}\left(t-\tau_{1}\right) \bar{\beta}\right) d t .
$$

Consequently, we have found control function (3.5) such that equalities (3.1)-(3.3) hold for all left-hand sides. It means complete controllability of $(2.3),(2.4)$ on $\left[\tau_{0}, \tau_{1}\right]$. Since the values of $\tau_{0}, \tau_{1} \subset T$ are arbitrary, so one can conclude that system (2.3), (2.4) is completely controllable on any set $\left[\tau_{0}, \tau_{1}\right] \subset T$. Therefore, it is differentially controllable on $T$ by Definition 5 .

Differential controllability condition can be formulated in terms of the controllability matrix

$$
\mathcal{S}(t)=\left(S_{0}(t) S_{1}(t) \ldots S_{n-d-1}(t)\right)
$$

where $S_{0}(t)=\mathcal{H}(t), \quad S_{i}(t)=J_{1}(t) S_{i-1}(t)-S_{i-1}^{\prime}(t), \quad i=\overline{1, n-d-1}$.

Theorem 3. Let us assume that

1) $A(t), B(t) \in \mathbf{C}^{2 r+n-d-1}(T), U(t), u(t) \in \mathbf{C}^{2 r+n-d}(T)$;

2) Assumptions 2) - 4) of Theorem 1 are satisfied.

System (1.1) is differentially controllable on $T$ if and only if the following conditions are satisfied: i) $\operatorname{rank} \mathcal{G}(t)=d \forall t \in T$;

ii) $\operatorname{rank} \mathcal{S}(t)=n-d$ for almost all $t$, i.e. for all points $t \in T$ except some sets with measure zero.

Proof. Sufficiency. Let us assume the opposite. Suppose that conditions i) and ii) are satisfied but system (1.1) is not differentially controllable on $T$. According to Theorem 2, it means that $h^{\top} X^{-1}(t) \mathcal{H}(t)=0$ on some interval $\left[\tau_{0}, \tau_{1}\right] \subset T$. Denote $F(t)=X^{-1}(t) \mathcal{H}(t)$. Taking into account that $\left(X^{-1}(t)\right)^{\prime}=J_{1}(t) X^{-1}(t)$ and sequentially differentiating matrix $F(t) n-d-1$ times, we obtain

$$
F(t)^{(j)}=(-1)^{j} X^{-1}(t) S_{j}(t),
$$

where $S_{j}$ are matrices from (3.12) and $F(t)^{(j)}=0 \forall t \in\left[\tau_{0}, \tau_{1}\right], \quad j=\overline{0, n-d-1}$.

Then $h^{\top} \mathcal{S}(t)=0 \forall t \in\left[\tau_{0}, \tau_{1}\right]$ for any nonzero $h \in \mathbf{R}^{n-d}$ so $\operatorname{rank} \mathcal{S}(t)<n-d$. We have the contradiction.

Necessity. Let system (1.1) be differentially controllable on T. According to Theorem 2:

a) condition i) of the Theorem is satisfied;

b) for any nonzero $h \in \mathbf{R}^{n-d}$ relation (3.4) is fulfilled for almost all $t \in T$.

Let us assume that in this case the rank of matrix $\mathcal{S}(t)<n-d$ on some interval $\left[\tau_{0}, \tau_{1}\right] \subset T$. Then, for any nonzero vector $p \in \mathbf{R}^{n-d}$ we have $p^{\top} \mathcal{S}(t)=0 \forall t \in\left[\tau_{0}, \tau_{1}\right]$ or

$$
p^{\top} S_{0}(t)=0, \quad p^{\top} S_{1}(t)=0, \quad \ldots, \quad p^{\top} S_{n-d-1}(t)=0 .
$$

Taken into account that $S_{0}(t)=\mathcal{H}(t)$, it follows from $(3.13)$ that $p^{\top} \mathcal{H}(t)=0$. Assuming that $p^{\top}=h^{\top} X^{-1}(t)$, we obtain the contradiction with (3.4). 


\subsection{Stationary systems}

In this case the original system has matrices with constant coefficients. Differential controllability conditions have a simple structure. Let us consider a stationary system of DAEs

$$
A x^{\prime}(t)=B x(t)+U u(t), \quad t \in I,
$$

where $A, B$ and $U$ are constant matrices with sizes $n \times n, n \times n$ and $n \times l$, respectively, $\operatorname{det} A=0$.

It is easy to see that the concepts of complete and differential controllability coincide for stationary systems. So, in this case, we deal with just controllability.

It is known [11] that in the case of a regular matrix pencil $\lambda A-B$ there exist invertible $(n \times n)$-matrices $P$ and $S$ such that

$$
P A S=\left(\begin{array}{cc}
O & N \\
E_{n-\sigma} & O
\end{array}\right), \quad P B S=\left(\begin{array}{cc}
O & E_{\sigma} \\
G & O
\end{array}\right),
$$

where $N$ is upper triangular matrix with $\rho$ square zero blocks on the diagonal so that $N^{\rho}=O$, $G$ is some square matrix of size $n-\sigma$.

It follows directly from [12] that the presence of the resolving minor in matrix $\mathrm{D}_{r, x}$ is a necessary and sufficient condition of existence of operator (2.2) in the case of constant coefficients. We show that there is such minor for $r=\rho$.

By left multiplying matrix $\mathrm{D}_{\rho, x}$ by the matrix $\operatorname{diag}\{P, \ldots, P\}$ and right multiplying by matrix $\operatorname{diag}\{S, \ldots, S\}$ and taking into account (3.15), we obtain

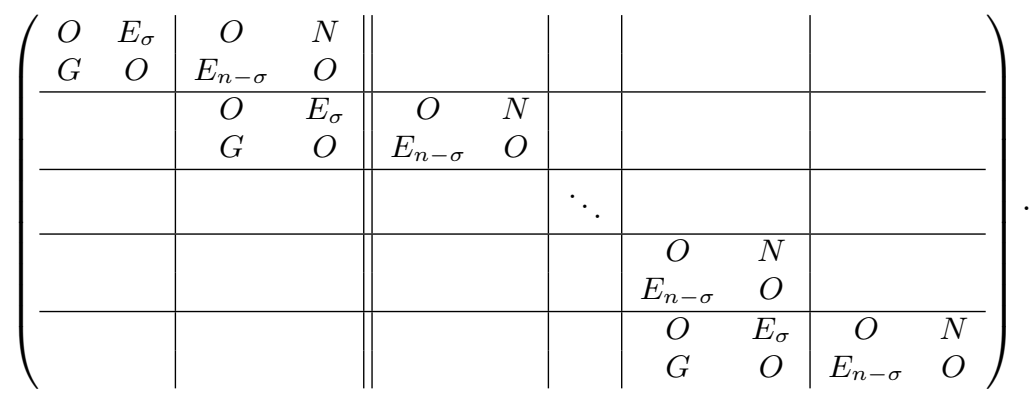

Obviously, that the rank of the matrix to the right of the double line in (3.16) is equal to the rank of matrix $\mathrm{D}_{\rho, z}$. Using the block transform of matrices, it is easy to show that $\operatorname{rank} \mathrm{D}_{\rho, z}=n \rho-\sigma$, since $N^{\rho}=O$. There is the resolving minor in matrix (3.16). It includes all columns of the blocks that contain the identity matrixes and $d=\sigma$. Thus, in the case of a regular matrix pencil $\lambda A-B$ there is the resolving minor in matrix $\mathrm{D}_{r, x}$ with $r=\rho$.

It is also easy to see that the relation

$$
\operatorname{rank}\left(\operatorname{diag}\{P, \ldots, P\} \mathrm{D}_{r+1, y} \operatorname{diag}\{S, \ldots, S\}\right)=\operatorname{rank}\left(\operatorname{diag}\{P, \ldots, P\} \mathrm{D}_{r, y} \operatorname{diag}\{S, \ldots, S\}\right)+n
$$

is satisfied with $r=\rho$.

Thus, in the case of a regular matrix pencil $\lambda A-B$ all the assumptions of Theorem 1 are fulfilled for DAE (3.14) with $r=\rho$. The equivalent form in this case is given by

$$
\begin{gathered}
x_{1}^{\prime}(t)=J_{1} x_{1}(t)+\mathcal{H} \bar{u}(t), \\
x_{2}(t)=J_{2} x_{1}(t)+\mathcal{G} \bar{u}(t), \quad t \in I,
\end{gathered}
$$

where

$$
\begin{gathered}
\operatorname{colon}\left(x_{1}(t), x_{2}(t)\right)=Q x(t), \quad x_{1}(t) \in \mathbf{R}^{n-d}, x_{2}(t) \in \mathbf{R}^{d} ; \\
\left(\begin{array}{c}
\mathcal{G} \\
\mathcal{H}
\end{array}\right)=\left(\begin{array}{lll}
G_{0} & \ldots & G_{r} \\
H_{0} & \ldots & H_{r}
\end{array}\right)=\left(\begin{array}{lll}
R_{0} U & \ldots & R_{r} U
\end{array}\right), \quad\left(\begin{array}{cc}
J_{2} & E_{d} \\
J_{1} & O
\end{array}\right)=R_{0} B Q^{-1} .
\end{gathered}
$$

Taking into account Theorems 2 and 3, we come to the following statements. 
Theorem 4. Let a matrix pencil $\lambda A-B$ be regular (i.e. $\operatorname{det}(\lambda A-B) \not \equiv 0)$. System (3.14) is controllable if and only if the following conditions are satisfied:

1) $\operatorname{rank} \mathcal{G}=d$;

2) $\forall h \in \mathbf{R}^{n-d}: h \neq 0, \quad h^{\top} X^{-1}(t) \mathcal{H} \not \equiv 0$.

Theorem 5. Let a matrix pencil $\lambda A-B$ be regular (i.e. $\operatorname{det}(\lambda A-B) \not \equiv 0$ ). System (3.14) is controllable if and only if the following conditions are satisfied:

1) $\operatorname{rank} \mathcal{G}=d$

2) $\operatorname{rank} \mathcal{Q}=n-d$.

Here $\mathcal{Q}=\left(\begin{array}{lllll}\mathcal{H} & J_{1} \mathcal{H} & \ldots & J_{1}^{n-d-1} \mathcal{H}\end{array}\right)$ is the controllability matrix of system $(3.17)$.

Conditions of Theorems 4 and 5 can be formulated in terms of input data for system (3.14), using the following substitutions

$$
J_{1}=\left(\begin{array}{ll}
O & E_{n-d}
\end{array}\right) R_{0} B Q^{-1}\left(\begin{array}{c}
E_{n-d} \\
O
\end{array}\right), \mathcal{H}=\left(\begin{array}{lll}
R_{0,2} U & \ldots & R_{r, 2} U
\end{array}\right), \quad\left(\begin{array}{c}
R_{i, 1} \\
R_{i, 2}
\end{array}\right)=R_{i}, \quad i=\overline{0, r} .
$$

Coefficients $R_{i}(i=\overline{0, r})$ are determined in terms of coefficients of DAE (3.14) and their derivatives defined in $(2.1),(2.6)$.

\section{Example}

Let us consider the linear system of DAE

$$
\begin{gathered}
\left(\begin{array}{ccc}
1+\cos t & 1-\cos t & 1 \\
-\sin t \cos t & 1-\sin t & -\sin t \\
0 & 0 & 0
\end{array}\right) x^{\prime}(t)+\left(\begin{array}{ccc}
-\cos t-\sin t & 1+\sin t+\cos ^{2} t & 0 \\
1+\sin ^{2} t & -\cos t & 0 \\
\cos t & 1 & 1
\end{array}\right) x(t)+ \\
+\left(\begin{array}{ll}
0 & 0 \\
1 & 0 \\
0 & 1
\end{array}\right) u(t)=0
\end{gathered}
$$

where $t \in I=[0,+\infty), \quad x(t): I \rightarrow \mathbf{R}^{3}$ is unknown function.

We investigate system (4.1) on differential controllability on $I$. To do this, we verify all the assumptions of Theorem 3 .

Condition 1) is obviously satisfied. To verify condition 2) we construct the matrices

$\mathrm{D}_{1, x}=\left(\begin{array}{cc}-P_{1}-P_{2} & 1+P_{2}+P_{1}^{2} \\ 1+P_{2}^{2} & -P_{1} \\ P_{1} & 1 \\ \hline P_{2}-P_{1} & P_{1}-\sin (2 t) \\ \sin (2 t) & P_{2} \\ -P_{2} & 0\end{array}\right.$

\begin{tabular}{|cccccc|}
\hline 0 & $1+P_{1}$ & $1-P_{1}$ & 1 & 0 & 0 \\
0 & $-P_{1} P_{2}$ & $1-P_{2}$ & $-P_{2}$ & 0 & 0 \\
1 & 0 & 0 & 0 & 0 & 0 \\
\hline 0 & $-P_{1}-2 P_{2}$ & $2 P_{2}+P_{1}^{2}+1$ & 0 & $1+P_{1}$ & $1-P_{1}$ \\
0 & $3 P_{2}^{2}$ & $-2 P_{1}$ & $-P_{1}$ & $-P_{1} P_{2}$ & $1-P_{2}$ \\
0 & $P_{1}$ & 1 & 1 & 0 & 0 \\
\hline
\end{tabular}

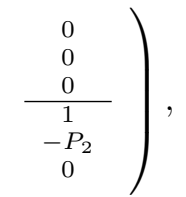

$\mathrm{D}_{2, y}=\left(\begin{array}{cc}1+P_{1} & 1-P_{1} \\ -P_{1} P_{2} & 1-P_{2} \\ 0 & 0 \\ -P_{1}-2 P_{2} & 2 P_{2}+P_{1}^{2}+1 \\ 3 P_{2}^{2} & -2 P_{1} \\ P_{1} & 1 \\ 2 P_{2}-3 P_{1} & 3 P_{1}-2 \sin (2 t) \\ 4 \sin (2 t) & 3 P_{2} \\ -2 P_{2} & 0\end{array}\right.$

\begin{tabular}{c|c}
1 & $\mid$ \\
$-P_{2}$ & $\mid$ \\
0 & $\mid$ \\
0 & $\mid$ \\
$-P_{1}$ & $\mid$ \\
1 & \\
0 & $\mid$ \\
$P_{2}$ & $\mid$ \\
0 &
\end{tabular}

0
0
0
$1+P_{1}$
$-P_{1} P_{2}$
0
$-P_{1}-2 P_{2}$
$3 P_{2}^{2}$
$P_{1}^{2}$

0
0
0
$1-P_{1}$
$1-P_{2}$
0
$P_{2}+P_{1}^{2}+1$
$-2 P_{1}$
1

\begin{tabular}{c|c}
0 & $\mid$ \\
0 & $\mid$ \\
0 & $\mid$ \\
1 & $\mid$ \\
$-P_{2}$ & $\mid$ \\
0 & $\mid$ \\
0 & 1 \\
$-P_{1}$ & 1 \\
1 &
\end{tabular}

$\left.\begin{array}{ccc}0 & 0 & 0 \\ 0 & 0 & 0 \\ 0 & 0 & 0 \\ 0 & 0 & 0 \\ 0 & 0 & 0 \\ 0 & 0 & 0 \\ 1+P_{1} & 1-P_{1} & 1 \\ -P_{1} P_{2} & 1-P_{2} & -P_{2} \\ 0 & 0 & 0\end{array}\right)$,

where $P_{1}=\cos t$ and $P_{2}=\sin t$.

It is easy to see that $\operatorname{rank} \mathrm{D}_{1, z}=\rho=2 \forall t \in I$. The columns that are included into the resolving minor are framed in matrix $\mathrm{D}_{1, x}$. It includes $\rho=2$ columns of matrix $\mathrm{D}_{1, z}, n=3$ 
first columns of matrix $\mathrm{D}_{1, y}$ and the third column of matrix $\mathrm{D}_{1, x}$. It is easy to verify that $\operatorname{rank} \mathrm{D}_{2, y}=\operatorname{rank} \mathrm{D}_{1, y}+n=8$.

Thus, the conditions are fulfilled wherein system (4.1) has an equivalent form. Let us find the coefficients of operator $(2.2)$

$$
\mathcal{R}=\left(\begin{array}{ccc}
1 & \cos t & 0 \\
0 & 1 & 0 \\
0 & 0 & 1
\end{array}\right)+\left(\begin{array}{ccc}
0 & 0 & \cos t \sin t-1 \\
0 & 0 & \sin t \\
0 & 0 & 0
\end{array}\right) \frac{d}{d t}
$$

that converts DAE (4.1) into the system

$$
\begin{gathered}
x_{1}^{\prime}(t)+\left(\begin{array}{cc}
0 & 1+\sin t \\
1 & -\cos t
\end{array}\right) x_{1}(t)+\left(\begin{array}{cc}
\cos t & 0 \\
1 & 0
\end{array}\right) u(t)+\left(\begin{array}{cc}
0 & \cos t \sin t-1 \\
0 & \sin t
\end{array}\right) u^{\prime}(t)=0 \\
x_{2}(t)+\left(\begin{array}{cc}
\cos t & 1
\end{array}\right) x_{1}(t)+\left(\begin{array}{ll}
0 & 1
\end{array}\right) u(t)=0 .
\end{gathered}
$$

Finally we obtain

$$
\mathcal{H}(t)=\left(\begin{array}{cccc}
\cos t & 0 & 0 & \cos t \sin t-1 \\
1 & 0 & 0 & \sin t
\end{array}\right), \quad \mathcal{G}(t)=\left(\begin{array}{cccc}
0 & 1 & 0 & 0
\end{array}\right)
$$

Then $\operatorname{rank} \mathcal{G}(t)=d=1$, i.e. condition i) of Theorem 3 is satisfied.

We construct the controllability matrix of system (4.3)

$$
\mathcal{S}(t)=\left(S_{0}(t) S_{1}(t)\right)
$$

where $S_{0}(t)=\mathcal{H}(t), S_{1}(t)=J_{1}(t) \mathcal{H}(t)-\mathcal{H}^{\prime}(t)$.

Then

$$
\mathcal{S}(t)=\left(\begin{array}{cccc}
1+2 \sin t & 0 & 0 & \sin t+3 \sin ^{2} t-1 \\
0 & 0 & 0 & -1-\cos t
\end{array}\right) .
$$

We have $\operatorname{rank} \mathcal{S}(t)=1$ when $1+2 \sin t=0$ and $-1-\cos t=0$, i.e. in the points $t=-\pi / 6+$ $2 \pi n, t=7 \pi / 6+2 \pi n, t=\pi+2 \pi n, n \in \mathbf{N}$. Thus, DAE (4.1) is not differentially controllable on any interval $T \subset I$, containing the points $t=-\pi / 6+2 \pi n, t=7 \pi / 6+2 \pi n, t=\pi+2 \pi n, n \in \mathbf{N}$. Alternatively, $\mathcal{S}(0)=\left(\begin{array}{cccc}1 & 0 & 0 & -1 \\ 0 & 0 & 0 & -2\end{array}\right)$ so $\operatorname{rank} \mathcal{S}(0)=2=n-d \forall t \in I$. According to Lemma 4 [10], system (4.1) is completely controllable on any interval $T \subset I$ that contain point $t=0$ and also points $t=-\pi / 6+2 \pi n, t=7 \pi / 6+2 \pi n, t=\pi+2 \pi n, n \in \mathbf{N}$.

This work was partially supported by the Russian Foundation for Basic Research (project no. 16-31-00101), by the Complex Program of Fundamental Scientific Research of Siberian Branch of RAS (no. II.2) and by the Council for Grants of the President of Russian Federation for state support of the leading scientific schools (project NSh-8081.2016.9).

\section{References}

[1] K.E.Brenan, S.L.Campbell, L.R.Petzold, Numerical solution of initial-value problems in differential-algebraic equations, SIAM, 1996.

[2] S.L.Campbell, E.Griepentrog, Solvability of general differential algebraic equations, SIAM J. Sci. Stat. Comp., 16(1995), 257-270.

[3] I.V.Gaishun, Introduction to the theory of linear nonstationary systems, Publishing Natsional'naya Akademiya Nauk Belarusi, Institut Matematiki, 1999 (in Russian). 
[4] V.Mehrmann, T.Stykel, Descriptor systems: a general mathematical framework for modelling, simulation and control, At-Automatisierungstechnik., 54(2006), no. 8, 405-415.

[5] E.L.Yip, R.F.Sincovec, Solvability, controllability and observability of continuous descriptor systems, IEEE Trans. Autom. Control., AC-26(1981), 702-707.

[6] L.Dai, Singular control system, Lecture notes in control and information sciences, 118, Springer-Verlag, Berlin, Heidelberg, N.Y., 1989.

[7] S.L.Campbell, N.K.Nichols, W.J.Terrell, Duality, observability, and controllability for linear time-varying descriptor systems Circ, Syst. and Sign. Process., 10(1991), 455-470.

[8] A.A.Shcheglova, P.S.Petrenko, The R-observability and R-controllability of linear differential-algebraic systems, Izv. Vyssh. Uchebn. Zaved., Mat., 2012, no. 3, 74-91 (in Russian).

[9] P.S.Petrenko, Local R-controllability to zero of nonlinear algebraic-differential systems, Izv. Irkutsk. Gos. Univ., Ser. Matemat., 4(2011), no. 4, 101-115.

[10] A.A.Shcheglova, Controllability of nonlinear algebraic differential systems, Avtom. Telemekh., 2008, no. 10, 57-80 (in Russian).

[11] F.R.Gantmacher, The theory of matrices, German, Matrizentheorie, Berlin, New York, Springer-Verlag, 1986.

[12] A.A.Shcheglova, The transformation of a linear algebraic-differential system to an equivalent form, Proceeding of the IX International Chetaev Conference «Analytical Mechanics, Stability and Motion Control», Irkutsk, June 2007, vol. 5, 298-307 (in Russian).

\section{Дифференциальная управляемость линейных систем дифференциально-алгебраических уравнений}

Павел С. Петренко

Институт динамики систем и теории управления имени В. М. Матросова СО РАН Лермонтова, 134, Иркутск, 664033

Россия

\footnotetext{
Рассматривается линейная система обыкновенных дифференииалъных уравнений с переменными коэффициентами, не разрешенная относительно производной искомой вектор-функиии и тождественно вырожденная в области определения. Допускается произвольно высокий индекс неразрешенности системы. Исследуется дифференииальная управляемость такой системы в предположениях, обеспечивающих существование эквивалентной в смысле решений структурной формы с разделенными "дифференциальной" и "алгебрачческой" подсистемами.

Ключевые слова: дифференциально-алгебрачческие уравнения, дифференциальная управляемость, полная управляемость.
} 